\title{
On solutions of the d'Alembert equation on a restricted domain
}

\author{
ANNA BAHYRYCZ AND JANUSZ BRZDȨK
}

Abstract. Let $A$ be a subgroup of a commutative group $(G,+)$ and $P$ be a commutative ring. We give the full description of functions $g: G \rightarrow P$ satisfying

$$
g(x+y)+g(x-y)=2 g(x) g(y) \quad(x, y) \in A \times G .
$$

Thus we obtain a family of functions depicting evolutions of quite arbitrary functions $g_{0}$ : $G \rightarrow P$ into cosine functions $g: G \rightarrow P$, i.e., solutions of the d'Alembert (cosine) functional equation

$$
g(x+y)+g(x-y)=2 g(x) g(y) \quad x, y \in G .
$$

We also show that every function $g: G \rightarrow P$, fulfilling (A), is a solution of (B) if and only if $A=G$.

Mathematics Subject Classification (2010). 39B52, 39B55.

Keywords. d'Alembert's functional equation, restricted domain, subgroup, commutative group, ring without zero divisors, lifting.

\section{Introduction}

Let $(H, \cdot)$ and $(F, \cdot)$ be groups. In the class of functions mapping $H$ into $F$, Dhombres and Ger $[10,11]$ investigated the equation of homomorphism on a restricted domain. Namely, they studied functions $h: H \rightarrow F$ satisfying

$$
h(x y)=h(x) h(y) \quad(x, y) \in Z,
$$

for some kinds of sets $Z \subset H^{2}$. In particular, they have proved the following (see [10, Théorème 1], [11, Theorems 1 and 2]).

Theorem 1. Let $Y$ be a nonempty subset of $H$ and $Z=H \times Y$. Assume that $F$ is of order greater than 2 . Then the following two statements are valid.

$(\alpha)$ A solution $h: H \rightarrow F$ of (1) is a homomorphism if and only if the subgroup generated by $Y$ is $H$. 
( $\beta)$ If $H$ is commutative, then $h: H \rightarrow F$ satisfies (1) if and only if

$$
h(x)=g\left(x \xi(\pi(x))^{-1}\right) \lambda(\pi(x)) \quad x \in H,
$$

for some $\lambda: H / H_{0} \rightarrow F$ with $\lambda(e)=e$ and some homomorphism $g$ : $G_{0} \rightarrow F$, where $H_{0}$ is the subgroup of $H$ that is generated by $Y$, e denotes the neutral elements in $H / H_{0}$ and $F, \pi: H \rightarrow H / H_{0}$ is the natural projection and $\xi: H / H_{0} \rightarrow H$ is a lifting (i.e., $\pi(\xi(u))=u$ for $u \in H / H_{0}$ ).

Actually, statement $(\beta)$ was proved in [11, Theorem 2] only for commutative $F$; however, it is easy to check that this assumption is not necessary.

In this paper we show that modifications of some classical reasonings allow us to obtain somewhat similar results for the d'Alembert functional equation

$$
g(x+y)+g(x-y)=2 g(x) g(y)
$$

in the class of functions mapping a commutative group $(G,+)$ into a commutative ring $(P,+, \cdot)$. However, since for the d'Alembert equation the reasonings are much more complicated than for the equation of homomorphism, we generally confine our investigation only to the situation in which (2) is valid for every $(x, y) \in A \times G$ with $A$ being a subgroup of $G$. In this way, changing the subgroup $A$, we obtain some families of functions (given in Theorem 2) that depict evolutions of quite arbitrary functions $g_{0}: G \rightarrow P$ (satisfying (2) for $y \in G$ and $x \in A=\{0\}$ ) into cosine functions, i.e., into functions $g: G \rightarrow P$ satisfying (2) for every $x, y \in G$ (the case of $A=G$ ).

The situation when (2) is valid for every $(x, y) \in G \times A$ is significantly different and needs a different reasoning. Therefore it will be considered in a separate paper.

Our results also correspond to [7, Theorems 1-4], where Corovei studied functions $g$ mapping a group $G$ into a skew field and satisfying one of the conditions:

$$
\begin{aligned}
g(x y)+g\left(x y^{-1}\right)=2 g(x) g(y) & (x, y) \in S \times G, \\
g(x y)+g\left(x y^{-1}\right)=2 g(x) g(y) & (x, y) \in G \times S
\end{aligned}
$$

with a subsemigroup $S$ of $G$, generating $G$ in some ways (e.g., $G=S \cup S^{-1}$ or $\left.G=S S^{-1}\right)$.

For more information on the d'Alembert equation, its generalizations, applications and related results see, e.g., [1-6,8,9,12-27] and the references therein.

Throughout this paper we assume that $P$ is a ring, $P \backslash\{0\} \neq \emptyset,(G,+)$ is a commutative group, and $A$ is a subgroup of $G$. For $a \in G, D \subset G$ we write $a+D:=\{a+b: b \in D\}, a-D:=\{a-b: b \in D\}$, and $2 D:=\{2 x: x \in D\}$.

\section{Auxiliary lemmas}

Let us begin with two auxiliary lemmas, concerning the case char $P \neq 2$, by which we mean that $-p \neq p$ for some $p \in P$. 
Lemma 1. Let $P$ be a commutative ring with unity denoted by 1 and without zero divisors, char $P \neq 2$, and

$(\mathcal{P})$ for every $q \in P \backslash\{0\}$ there is $p \in P$ with $p^{2} q=1$.

Assume that $g: G \rightarrow P$ satisfies

$$
g(x+y)+g(x-y)=2 g(x) g(y) \quad(x, y) \in A \times G .
$$

Then one of the following three statements is valid.

(i) $g(x)^{2}=1$ for $x \in A$.

(ii) $g(x)=0$ for $x \in A$.

(iii) There is a solution $m: G \rightarrow P$ of the equation

$$
m(x+y)=m(x) m(y) \quad(x, y) \in A \times G
$$

such that

$$
g(x)=\frac{1}{2}(m(x)+m(-x)) \quad x \in G .
$$

Proof. Let us start with a simple comment concerning (5). Namely, in view of $(\mathcal{P})$, there is $d \in P$ with $2 d=d+d=d 2=1$. Such $d$ is unique, because $P$ has no zero divisors. We denote that element $d$ by $\frac{1}{2}$.

Suppose that there exists $a \in A$ such that $g(a)^{2} \neq 1$. Take $k \in P$ with $4 k^{2}\left(g(a)^{2}-1\right)=1$. Putting $y=0$ in (3) we get $g(x)+g(x)=2 g(x) g(0)$ for $x \in A$, whence $2 g(x)(1-g(0))=0$ for $x \in A$. Hence $g(0)=1$ or $g(A)=\{0\}$.

Clearly it remains to consider the situation when $g(0)=1$. Then taking $x=0$ in $(3)$ we get $g(y)+g(-y)=2 g(y)$ for $y \in G$, which means that

$$
g(-y)=g(y) \quad y \in G
$$

Next, with $y=x=a$ in (3) we obtain $g(2 a)=2 g(a)^{2}-1$, whence

$$
2 k^{2}(g(2 a)-1)=4 k^{2}\left(g(a)^{2}-1\right)=1 .
$$

Define functions $h: G \rightarrow P$ and $m: G \rightarrow P$ by

$$
\begin{aligned}
h(x) & :=g(a+x)-g(a-x) \quad x \in G, \\
m(x) & :=g(x)+k h(x) \quad x \in G .
\end{aligned}
$$


We show that $m$ satisfies (4). So take $x \in A, y \in G$. Then (1) and (6)-(9) imply that

$$
\begin{aligned}
m(x) m(y)= & g(x) g(y)+k[h(x) g(y)+g(x) h(y)]+k^{2} h(x) h(y) \\
= & g(x) g(y)+k[g(a+x) g(y)-g(a-x) g(y)+g(x) g(a+y) \\
& -g(x) g(a-y)]+k^{2}[g(a+x) g(a+y)-g(a-x) g(a+y) \\
& -g(a+x) g(a-y)+g(a-x) g(a-y)] \\
= & \frac{1}{2}[g(x+y)+g(x-y)]+\frac{k}{2}[g(a+x+y)+g(a+x-y) \\
& -g(a-x+y)-g(a-x-y)+g(a+x+y)+g(a-x+y) \\
& -g(a+x-y)-g(a-x-y)]+\frac{k^{2}}{2}[g(2 a+x+y)+g(x-y) \\
& -g(2 a-x+y)-g(x+y)-g(2 a+x-y)-g(x+y) \\
& +g(2 a-x-y)+g(x-y)] \\
= & \frac{1}{2} g(x+y)+\frac{1}{2} g(x-y)+\frac{k}{2}[2 g(a+x+y)-2 g(a-x-y)] \\
& +\frac{k^{2}}{2}[2 g(2 a) g(x+y)-2 g(2 a) g(x-y)-2 g(x+y)+2 g(x-y)] \\
= & {\left[\frac{1}{2}+k^{2}(g(2 a)-1)\right] g(x+y)+\left[\frac{1}{2}-k^{2}(g(2 a)-1)\right] g(x-y) } \\
& +k h(x+y)=g(x+y)+k h(x+y)=m(x+y) .
\end{aligned}
$$

Observe that from conditions (6) and (8) we obtain

$$
h(-x)=-h(x) \quad x \in G,
$$

so by (9)

$$
m(-x)=g(x)-k h(x) \quad x \in G .
$$

Adding the above equality and (9) we obtain (5).

Let us recall that, if $S$ is a subgroup of $G$, then $\xi: G / S \rightarrow G$ is a lifting provided

$$
\xi([y]) \in[y] \quad y \in G,
$$

where $[y]:=y+S$ for $y \in G$. In what follows the neutral element in the factor group $G / S$ is also denoted by 0 .

Lemma 2. Assume that $P$ has the unity denoted by 1 , has no zero divisors, char $P \neq 2,(\mathcal{P})$ holds, $A$ is a subgroup of $G, g: G \rightarrow P$ and

$$
g(A) \subset\{-1,1\} .
$$

Then $g$ is a solution of Eq. (3) if and only if there exist a solution $\mu: A \rightarrow$ $\{-1,1\}$ of the equation

$$
\mu(x+y)=\mu(x) \mu(y) \quad x, y \in A,
$$


a lifting $\xi: G / A \rightarrow G$, a family of additive functions $\mathcal{L}_{\sigma}: A \rightarrow P$ for $\sigma \in G / A$, and a function $\kappa: G / A \rightarrow P$ such that

$$
\begin{gathered}
\xi(0)=0, \quad \xi([-y])=-\xi([y]) \quad y \in G,[y] \neq[-y], \\
\mathcal{L}_{[-y]}=-\mathcal{L}_{[y]} \quad y \in G, 2 y \notin \mu^{-1}(\{-1\}), \\
\kappa([y])=\frac{1}{2} \mathcal{L}_{[y]}(2 \xi([y])) \quad y \in G, 2 y \in \mu^{-1}(\{-1\}), \\
\kappa(0)=1, \quad \kappa([-y])=\kappa([y]) \quad y \in G,
\end{gathered}
$$

and

$$
g(v)=\mu(v-\xi([v]))\left(\kappa([v])+\mathcal{L}_{[v]}(v-\xi([v]))\right) \quad v \in G .
$$

Proof. First assume that $g$ is a solution of Eq. (3).

Take $x, y \in A$. If $g(x) g(y)=1$, then by $(3) g(x+y)+g(x-y)=2$ and consequently $g(x+y)=g(x-y)=1$. Hence $g(x+y)=1=g(x) g(y)$.

If $g(x) g(y)=-1$, then (3) yields $g(x+y)+g(x-y)=-2$, whence $g(x+y)=$ $g(x-y)=-1$. This means that $g(x+y)=-1=g(x) g(y)$, too.

Thus we have shown that the function $\mu: A \rightarrow\{-1,1\}, \mu(x)=g(x)$ for $x \in A$, is a solution of (10). In particular we get $\mu(2 x)=\mu(x)^{2}$ for $x \in A$ and consequently

$$
\mu(2 A)=g(2 A)=\{1\}
$$

which means that

$$
g(0)=1 .
$$

Hence (3) (with $x=0$ ) implies that

$$
g(y)=g(-y) \quad y \in G .
$$

Write $D:=\mu^{-1}(\{1\})$ and

$$
T_{y}(z):=g(z+y)-g(y) \quad z \in D, y \in G .
$$

Then $D$ is a subgroup of $A$ and

$$
g(z+y)=\mu(y)\left(g(y)+T_{y}(z)\right) \quad z \in D, y \in G .
$$

Moreover, (16) implies that

$$
2 A \subset D
$$

and, by (3) and (18),

$$
\begin{aligned}
T_{y}(w+z)+T_{y}(w-z)= & g(w+z+y)-g(y) \\
& +g(w-z+y)-g(y) \\
= & g(z+w+y)+g(z-w-y)-2 g(y) \\
= & 2 g(z) g(w+y)-2 g(y)=2 T_{y}(w)
\end{aligned}
$$

for every $w, z \in D, y \in G$. So we have proved that

$$
T_{y}(w+z)+T_{y}(w-z)=2 T_{y}(w) \quad w, z \in D, y \in G .
$$


Clearly (21) with $z=w$ yields

$$
T_{y}(2 z)=2 T_{y}(z) \quad z \in D, y \in G,
$$

because (in view of the definition of $\left.T_{y}\right) T_{y}(0)=0$ for $y \in G$.

Take $u, v \in D, y \in G$ and write $w=u+v$ and $z=u-v$. Then $w+z=$ $2 u, w-z=2 v \in D$ and consequently, by (21) and (22),

$$
\begin{aligned}
T_{y}(u+v) & =T_{y}(w)=\frac{1}{2}\left(T_{y}(w+z)+T_{y}(w-z)\right) \\
& =\frac{1}{2}\left(T_{y}(2 u)+T_{y}(2 v)\right)=T_{y}(u)+T_{y}(v) .
\end{aligned}
$$

Thus we have shown that $T_{y}$ is additive for each $y \in G$. Moreover, by (18),

$$
\begin{aligned}
T_{-y}(x) & =g(x-y)-g(-y)=g(y-x)-g(y) \\
& =T_{y}(-x)=-T_{y}(x) \quad x \in D, y \in G .
\end{aligned}
$$

In view of (20) we may define $L_{y}: A \rightarrow P$ by

$$
L_{y}(x):=\frac{1}{2} T_{y}(2 x) \quad x \in A .
$$

Note that, for every $x, z \in A, y \in G$,

$$
L_{y}(x+z)=\frac{1}{2} T_{y}(2 x+2 z)=\frac{1}{2} T_{y}(2 x)+\frac{1}{2} T_{y}(2 z)=L_{y}(x)+L_{y}(z),
$$

which means that $L_{y}$ is additive. Next, (23) yields

$$
\begin{aligned}
L_{-y}(x) & =\frac{1}{2} T_{-y}(2 x)=-\frac{1}{2} T_{y}(2 x) \\
& =-L_{y}(x) \quad x \in A, y \in G .
\end{aligned}
$$

Note also that replacing $x$ with $z$ and $y$ with $y+z$ in (3), for every $y \in$ $G, z \in A \backslash D$ we have

$$
g(y+2 z)+g(y)=2 g(z) g(y+z)=-2 g(z+y),
$$

which gives

$$
\begin{aligned}
g(y+z) & =-\frac{1}{2}(g(y+2 z)+g(y)) \\
& =-\frac{1}{2} T_{y}(2 z)-g(y)=\mu(z)\left(L_{y}(z)+g(y)\right) .
\end{aligned}
$$

Consequently, in view of (19) we have

$$
g(z+y)=\mu(z)\left(g(y)+L_{y}(z)\right) \quad y \in G, z \in A .
$$

Let $\xi: G / A \rightarrow G$ be a lifting such that

$$
\begin{gathered}
\xi(0)=0, \quad \xi([-y])=-\xi([y]) \quad y \in G, \quad[y] \neq[-y], \\
g(\xi([y])) \neq 0 \quad y \in G,[y]=[-y], g([y]) \neq\{0\} .
\end{gathered}
$$


Clearly, condition (26) is easy to obtain; next, if $y \in G \backslash A,[y]=[-y]$ and there exists $z \in[y]$ such that $g(z) \neq 0$, then we write $\xi([y])=z$, which guarantees (27).

\section{Taking}

$$
\mathcal{L}_{[u]}:=L_{\xi([u])}, \quad \kappa([u]):=g(\xi([u])) \quad u \in G,
$$

by (18) and (26) we get

$$
\begin{gathered}
\kappa(0)=g(\xi(0))=g(0)=1, \\
\kappa([-y])=g(\xi([-y]))=g(-\xi([y]))=g(\xi([y]))=\kappa([y]) \quad y \in G,
\end{gathered}
$$

and, on account of the definition of $\mu$ and (25) (with $y=\xi([w]$ ) and $z=$ $w-\xi([w]))$,

$$
\begin{aligned}
g(w) & =g(w-\xi([w])+\xi([w])) \\
& =\mu(w-\xi([w]))\left(g(\xi([w]))+L_{\xi([w])}(w-\xi([w]))\right) \\
& =\mu(w-\xi([w]))\left(\kappa([w])+\mathcal{L}_{[w]}(w-\xi([w]))\right) \quad w \in G,
\end{aligned}
$$

which give (14) and (15). Moreover, by (24) and (26) we get

$$
\mathcal{L}_{[-u]}=L_{\xi([-u])}=L_{-\xi([u])}=-L_{\xi([u])}=-\mathcal{L}_{[u]} \quad u \in G,[u] \neq[-u] .
$$

Fix $x \in A$ and $y \in G$ with $2 y \in A$ and $\mu(2 y)=g(2 y)=1$, i.e., $[-y]=[y]$ and $2 y \in D=\mu^{-1}(\{1\})$. Then

$$
[x+y]=[x-y]=[y]=[-y]
$$

and consequently, by (10), (18) and the definition of $\mu$,

$$
\begin{aligned}
\mu(x-y-\xi([x-y])) & =\mu(x) \mu(-2 y) \mu(y-\xi([y])) \\
& =\mu(x+y-\xi([x+y])) \\
& =\mu(x) \mu(y-\xi([y])) .
\end{aligned}
$$

Hence, in view of the additivity of $\mathcal{L}_{[y]}$ and by (15),

$$
\begin{aligned}
g(x+y)+g(x-y)= & \mu(x+y-\xi([x+y]))(\kappa([x+y]) \\
& \left.+\mathcal{L}_{[x+y]}(x+y-\xi([x+y]))\right) \\
& +\mu(x-y-\xi([x-y]))(\kappa([x-y]) \\
& +\mathcal{L}_{[x-y]}(x-y-\xi([x-y])) \\
= & \mu(x) \mu(y-\xi([y]))(2 \kappa([y]) \\
& +\mathcal{L}_{[y]}(x+y-\xi([y])) \\
& \left.+\mathcal{L}_{[y]}(x+y-\xi([y]))+\mathcal{L}_{[y]}(-2 y)\right) \\
= & \mu(x) \mu(y-\xi([y]))\left(2 \kappa([y])+2 \mathcal{L}_{[y]}(y-\xi([y]))\right. \\
& \left.+2 \mathcal{L}_{[y]}(x)-\mathcal{L}_{[y]}(2 y)\right)
\end{aligned}
$$


and

$$
2 g(x) g(y)=2 \mu(x) \mu(y-\xi([y]))\left(\kappa([y])+\mathcal{L}_{[y]}(y-\xi([y]))\right) .
$$

So, (3) yields $2 \mathcal{L}_{[y]}(x)-\mathcal{L}_{[y]}(2 y)=0$.

Thus we have shown that $\mathcal{L}_{[y]}(x)=\frac{1}{2} \mathcal{L}_{[y]}(2 y)$ for every $x \in A, y \in G$ with $[y]=[-y]$ and $\mu(2 y)=1$, which means that in this case $\mathcal{L}_{[y]}$ is constant, and in view of the additivity of $\mathcal{L}_{[y]}$ we obtain $\mathcal{L}_{[y]}(x)=0$ for $x \in A$, which jointly with (28) gives (12).

Fix $x \in A$ and $y \in G$ with $2 y \in A$ and $\mu(2 y)=g(2 y)=-1$, i.e., $[-y]=[y]$ and $2 y \in A \backslash D$. Then by (10) and (16) we have

$$
g(-2 \xi([y]))=\mu(-2 \xi([y]))=\mu(2(y-\xi([y]))) \mu(-2 y)=-1 .
$$

Hence and from (3) and (18) we get

$$
\begin{aligned}
\mathcal{L}_{[y]}(2 \xi([y])) & =L_{\xi([y])}(2 \xi([y]))=\frac{1}{2} T_{\xi([y])}(4 \xi([y])) \\
& =\frac{1}{2}(g(5 \xi([y]))-g(\xi([y]))+(g(-\xi([y])-g(\xi([y])))) \\
& =\frac{1}{2}(2 g(2 \xi([y])) g(3 \xi([y]))-2 g(\xi([y]))) \\
& =-(g(3 \xi([y]))+g(\xi([y])))=-(2 g(2 \xi([y])) g(\xi([y]))) \\
& =2 g(\xi([y]))=2 \kappa([y]) .
\end{aligned}
$$

This completes the proof of the necessary condition.

Now assume that $g$ has the form described in the statement. Then (12) implies that

$$
\mathcal{L}_{[y]}(x)=0 \quad x \in A, \quad y \in G, \quad[y]=[-y], \quad \mu(2 y)=1 .
$$

Moreover, since $\kappa(0)=1$ and $\xi(0)=0$, we get

$$
g(x)=\mu(x-\xi([x]))\left(\kappa([x])+\mathcal{L}_{[x]}(x-\xi([x]))\right)=\mu(x) \quad x \in A .
$$

It is easy to check that, by (10),

$$
g(x+y)+g(x-y)=2 g(x) g(y) \quad x, y \in A .
$$

Take $x \in A$ and $y \in G \backslash A$. Clearly

$$
[x+y]=[y]=[y-x] .
$$

If $[y]=[-y]$, then (29) and (30) imply that, in the case $\mu(2 y)=1$,

$$
\begin{aligned}
g(x+y)+g(x-y)= & \mu(x+y-\xi([x+y])) \kappa([x+y]) \\
& +\mu(x-y-\xi([x-y])) \kappa([x-y]) \\
= & \mu(x) \mu(y-\xi([y])) 2 \kappa([y]) \\
= & 2 \mu(x) g(y)=2 g(x) g(y),
\end{aligned}
$$


and, in the case $\mu(2 y)=-1$,

$$
\begin{aligned}
g(x+y)+g(x-y)= & \mu(x+y-\xi([x+y]))(\kappa([x+y]) \\
& \left.+\mathcal{L}_{[x+y]}(x+y-\xi([x+y]))\right) \\
& +\mu(x-y-\xi([x-y]))(\kappa([x-y]) \\
& \left.+\mathcal{L}_{[x-y]}(x-y-\xi([x-y]))\right) \\
= & \mu(x) \mu(y-\xi([y]))\left(\kappa([y])+\mathcal{L}_{[y]}(x+y-\xi([y]))\right. \\
& +\mu(x) \mu(y-\xi([y]) \mu(-2 y))(\kappa([y]) \\
& \left.+\mathcal{L}_{[y]}(x-y-\xi([y]))\right) \\
= & \mu(x) \mu(y-\xi([y]))\left(\mathcal{L}_{[y]}(2 y)\right) \\
= & g(x) \mu(y-\xi([y]))\left(\mathcal{L}_{[y]}(2 \xi([y])\right. \\
& \left.+\mathcal{L}_{[y]}(2 y-2 \xi([y]))\right) \\
= & g(x) \mu(y-\xi([y]))\left(2 \kappa([y])+2 \mathcal{L}_{[y]}(y-\xi([y]))\right) \\
= & 2 g(x) g(y) .
\end{aligned}
$$

Moreover, if $[y] \neq[-y]$, then by (10)-(12) and (30), we get

$$
\begin{aligned}
g(x+y)+g(x-y)= & \mu(x+y-\xi([x+y]))(\kappa([x+y]) \\
& \left.+\mathcal{L}_{[x+y]}(x+y-\xi([x+y]))\right) \\
& +\mu(x-y-\xi([x-y]))(\kappa([x-y]) \\
& \left.+\mathcal{L}_{[x-y]}(x-y-\xi([x-y]))\right) \\
= & \mu(x) \mu(y-\xi([y]))(2 \kappa([y]) \\
& \left.+\mathcal{L}_{[y]}(x+y-\xi([y]))+\mathcal{L}_{[y]}(y-x-\xi([y]))\right) \\
= & 2 \mu(x) \mu(y-\xi([y]))\left(\kappa([y])+\mathcal{L}_{[y]}(y-\xi([y]))\right) \\
= & 2 \mu(x) g(y)=2 g(x) g(y) .
\end{aligned}
$$

Remark 1 . Note that actually in Lemma 2 hypothesis $(\mathcal{P})$ can be replaced by the following much weaker condition: there exists $d \in P$ with $2 d=1$. Clearly, such an element $d$ we can denote by $\frac{1}{2}$.

Remark 2. Conditions (11)-(14) look complicated, but actually they are not. Namely, we first choose $\xi$ and the family of functions $\mathcal{L}_{[x]}$; conditions (11) and (12) seem to be quite easy. Further, (13) determines uniquely the value of $\kappa$ for every $y \in G$ with $2 y \in \mu^{-1}(\{-1\})$. Clearly, if $y-(-y)=2 y \in \mu^{-1}(\{-1\}) \subset A$, then $[y]=[-y]$ and $\kappa([y])=\kappa([-y])$. Therefore condition (13) is not restricted by (14) in any way.

Remark 3. Assume that $A$ is divisible by 2 (i.e., for each $x \in A$ there is $z \in A$ with $x=2 z$ ). Then each solution $\mu: A \rightarrow\{-1,1\}$ of Eq. (10) can 
take only the value 1 , i.e., $\mu^{-1}(\{-1\})=\emptyset$ and consequently the form of solutions $g: G \rightarrow P$ of (3), described in Lemma 2, can be significantly simplified. Namely, $g: G \rightarrow P$ is a solution of Eq. (3) if and only if there exist a lifting $\xi: G / A \rightarrow G$, a family of additive functions $\mathcal{L}_{\sigma}: A \rightarrow P$ for $\sigma \in G / A$, and a function $\kappa: G / A \rightarrow P$ such that (11) and (15) are valid and

$$
\begin{gathered}
\mathcal{L}_{[-y]}=-\mathcal{L}_{[y]} \quad y \in G, \\
\kappa(0)=1, \kappa([-y])=\kappa([y]) \quad y \in G .
\end{gathered}
$$

Remark 4. Note that condition (15) can be written in the following equivalent form: $g(\xi(\sigma)+a)=\mu(a)\left(\kappa(\sigma)+\mathcal{L}_{\sigma}(a)\right)$ for $\sigma \in G / A, a \in A$.

\section{The main results in the case char $P \neq 2$}

The next theorem describes the general solution of (3) in the case when char $P \neq 2$.

Theorem 2. Assume that $P$ is a commutative ring with unity denoted by 1 and without zero divisors, $(\mathcal{P})$ holds, char $P \neq 2$, and $A$ is a subgroup of $G$. Then $g: G \rightarrow P$ is a solution of equation (3) if and only if one of the following three statements is valid.

(a) There exists a mapping $\gamma: G / 2 A \rightarrow P$ such that

$$
\begin{aligned}
\gamma([-u]) & =-\gamma([u]) \quad u \in G, \\
\gamma([x]) & =0 \quad x \in A,
\end{aligned}
$$

and

$$
g(y)=\gamma([y]) \quad y \in G .
$$

(b) $g$ has the form described in Lemma 2.

(c) There are a function $h: G / A \rightarrow P$ with $h(0)=1$, a solution $s: A \rightarrow P$ of the equation

$$
s(x+y)=s(x) s(y) \quad x, y \in A,
$$

and a lifting $\xi: G / A \rightarrow G$ with $\xi(0)=0$ such that (5) holds with $m$ : $G \rightarrow P$ given by

$$
m(y)=s(y-\xi([y])) h([y]) \quad y \in G .
$$

Proof. Let $g: G \rightarrow P$ be a solution of Eq. (3). On account of Lemma 1, one of statements $(i)-($ iii $)$ is valid.

Assume first that $g(A)=\{0\}$. Then (3) yields

$$
g(x+y)+g(x-y)=2 g(x) g(y)=0 \quad x \in A, y \in G,
$$

whence with $x=0$ we get

$$
g(y)=-g(-y) \quad y \in G,
$$


and consequently, with $y$ replaced by $x+y$ in (35), we deduce that

$$
g(2 x+y)=-g(-y)=g(y) \quad x \in A, y \in G .
$$

So, we may define a function $\gamma: G / 2 A \rightarrow P$ by $\gamma([y])=g(y)$ for $y \in G$. It is easily seen that $\gamma(x)=0$ for $x \in A, \gamma(-v)=-\gamma(v)$ for $v \in G / 2 A$ and $g(x+y)=\gamma([y])$ for $x \in 2 A, y \in G$. Thus we obtain $(a)$.

If $g(x) \in\{-1,1\}$ for $x \in A$, then we apply Lemma 2. So, it remains to consider the case where $g(A) \neq\{0\}$ and $g(a)^{2} \neq 1$ for some $a \in A$. Then statement (iii) of Lemma 1 must be valid. Moreover, according to Theorem $1(\beta), m$ has form (34).

Now we show the sufficient condition. First, let $g$ be the function described by $(a)$. Take $y \in G$ and $x \in A$. Then $(x+y)-(y-x)=2 x \in 2 A$, which means that $[x+y]=[y-x]$, where $[z]:=z+2 A$ for $z \in G$. Hence

$$
g(x+y)=\gamma([x+y])=\gamma([y-x])=-\gamma([x-y])=-g(x-y) .
$$

Consequently

$$
g(x+y)+g(x-y)=0=2 \gamma([x]) \gamma([y])=2 g(x) g(y) .
$$

If $(b)$ is valid, then it is enough to use Lemma 2.

Finally, assume that $g$ has the form described by $(c)$, i.e., (5) holds with $m: G \rightarrow P$ given by (34). Then, by Theorem $1(\beta), m$ satisfies (4). Consequently, for every $x \in A, y \in G$, we have

$$
\begin{aligned}
g(x+y)+g(x-y)= & \frac{1}{2}[m(x+y)+m(-x-y) \\
& +m(x-y)+m(-x+y)] \\
= & \frac{1}{2}[m(x) m(y)+m(-x) m(-y) \\
& +m(x) m(-y)+m(-x) m(y)] \\
= & \frac{1}{2}(m(x)+m(-x))(m(y)+m(-y)) \\
= & 2 g(x) g(y) .
\end{aligned}
$$

Thus we have proved that, in each of the cases $(a)-(c), g$ is a solution to (3).

The next proposition corresponds to statement $(\alpha)$ of Theorem 1.

Proposition 1. Assume that $A$ is a subgroup of $G, P$ has the unit element denoted by 1 , and char $P \neq 2$. Then every solution $g: G \rightarrow P$ of (3) satisfies the d'Alembert equation

$$
g(x+y)+g(x-y)=2 g(x) g(y) \quad x, y \in G
$$

if and only if $A=G$. 
Proof. Clearly, only the necessary condition needs a justification. So, suppose that $A \neq G$. Let $g: G \rightarrow P$ be given by

$$
g(A)=\{1\}, \quad g(G \backslash A)=\{0\} .
$$

It is easy to see that $g$ is a solution to (3) (it is described by statement $(b)$ of Theorem 2, i.e., in Lemma 2). However, for every $v \in G \backslash A$ we have

$$
g(v+v)+g(v-v)=g(2 v)+g(0) \in\{1,2\},
$$

but $2 g(v)^{2}=0$, which means that $g$ is not a solution to (36).

\section{The main results in the case char $P=2$}

We end the paper with a theorem and a proposition concerning the situation when $-p=p$ for $p \in P$, which we denote shortly by char $P=2$, though we do not assume that $P$ is without zero divisors. They complement Theorem 2 and Proposition 1. Since in this case reasonings are much simpler, we consider a more general situation where $A$ is not necessarily a subgroup of $G$.

Theorem 3. Let char $P=2, A \neq \emptyset$ and $2 a \in A$ for some $a \in A$. Then $g: G \rightarrow P$ satisfies (3) if and only if there exits a mapping $\gamma: G / S_{0} \rightarrow P$ such that

$$
\gamma([y])=\gamma([-y]) \quad \text { and } \quad g(y)=\gamma([y]) \quad y \in G,
$$

where $S_{0}$ denotes the subgroup of $G$ that is generated by the set $2 A$.

Proof. Since $2 p=0$ for $p \in P$, condition (3) takes the following form

$$
g(x+y)+g(x-y)=0 \quad x \in A, y \in G .
$$

Assume first that $g$ is a solution of (38). Replacing $y$ with $y+a$ and then $y$ with $y-a$ in (3) we get

$$
\begin{aligned}
& g(2 a+y)+g(-y)=0, \\
& g(2 a-y)+g(y)=0
\end{aligned}
$$

for every $y \in G$. Adding the above equalities and using (38) we have

$$
g(2 a+y)+g(2 a-y)=0=g(-y)+g(y),
$$

which yields

$$
g(y)=-g(-y)=g(-y) \quad y \in G,
$$

because $p=-p$ for $p \in P$. Further, replacing $y$ by $y+x$ in (38) we obtain $g(2 x+y)=g(y)$ for $y \in G, x \in A$. Thus we have proved that

$$
g(2 x+y)=g(y)=g(-y) \quad x \in A, y \in G .
$$

Let

$$
A_{0}:=\{x \in G: g(x+y)=g(y) \text { for } y \in G\} .
$$


Take $x, z \in A_{0}$. Then, for every $y \in G$,

$$
g(x-z+y)=g(-z+y)=g(z-z+y)=g(y),
$$

which means that $x-z \in A_{0}$. In this way we have shown that $A_{0}$ is a subgroup of the group $G$.

Let $S_{0}$ denote the subgroup of $G$ that is generated by $2 A$. Clearly, in view of (39), $2 A \subset A_{0}$, whence $S_{0} \subset A_{0}$. Consequently

$$
g(x+y)=g(y) \quad x \in S_{0}, y \in G .
$$

So, we may define a function $\gamma: G / S_{0} \rightarrow P$ by $\gamma([y])=g(y)$ for $y \in G$. It is easily seen that, by (39) and (40), condition (37) is fulfilled.

To complete the proof, suppose that $\gamma: G / S_{0} \rightarrow P$ is a mapping such that (37) holds, where $S_{0}$ denotes the subgroup of $G$ that is generated by the set $2 A$. Take $x \in A$ and $y \in G$. Then $x+y-(y-x)=2 x \in 2 A \subset S_{0}$, which means that $[x+y]=[y-x]$ and consequently, by $(37)$,

$$
\begin{aligned}
g(x+y)+g(x-y) & =\gamma([x+y])+\gamma([x-y]) \\
& =\gamma([x+y])+\gamma([y-x])=2 \gamma([x+y]) \\
& =0=2 \gamma([x]) \gamma([y])=2 g(x) g(y) .
\end{aligned}
$$

Thus we have proved that $g$ is a solution to (3).

Proposition 2. Let char $P=2, A \neq \emptyset$ and $2 a \in A$ for some $a \in A$. Every solution $g: G \rightarrow P$ of (3) satisfies the d'Alembert equation (36) if and only if the subgroup of $G$ that is generated by $2 A$ is equal to $2 G$.

Proof. Let $S_{0}$ denote the subgroup generated by $2 A$. First assume that $S_{0}=$ $2 G$. Let $g: G \rightarrow P$ be a solution to (3). Then $g$ has the form described in Theorem 3. Since, for every $z, y \in G$, we have $z+y-(z-y)=2 y \in 2 G=S_{0}$, this means that $g(z+y)=g(z-y)$ and consequently

$$
g(z+y)+g(z-y)=2 g(z+y)=0=2 g(z) g(y) .
$$

Now, suppose that there is $z \in 2 G \backslash S_{0}$. Then $z=2 v$ for some $v \in G$. Take $p \in P$ with $p \neq 0$ and define $\gamma: G / S_{0} \rightarrow P$ by $\gamma([z])=\gamma([-z])=p$ and $\gamma([y])=0$ for $y \in G$ with $[-z] \neq[y] \neq[z]$. Let $g(y)=\gamma([y])$ for $y \in G$. Then, by Theorem $3, \mathrm{~g}$ is a solution to (3), but

$$
g(v+v)+g(v-v)=g(2 v)+g(0)=p \neq 0=2 g(v) g(v) .
$$

Remark 5. In general the statement that the subgroup of $G$ that is generated by $2 A$ is equal to $2 G$ is not equivalent to the condition: the subgroup of $G$ that is generated by $A$ is equal to $G$. Namely, let $G$ be the multiplicative group of the nonzero real numbers and $A$ be a subset of $(0, \infty)$ such that the subgroup of $G$ that is generated by $2 A$ equals $(0, \infty)$ (for instance this is the case when 
$A$ is of positive outer Lebesgue measure or contains a subset of the second category and with the Baire property (cf., e.g., [11])). Then clearly the subgroup of $G$ that is generated by $A$ is not $G$.

\section{Open problems}

In connection with Theorems 1 and 3 and Proposition 2 the following natural question comes into mind: can we weaken the assumption that $A$ is a subgroup of $G$ in the case char $P \neq 2$ ? Moreover, what can be said in this case about solutions to (3) without any of the following assumptions:

(a) commutativity of $G$;

(b) commutativity of $P$;

(c) no zero divisors in $P$;

$(\mathrm{d})$ hypothesis $(\mathcal{P})$ ?

Open Access. This article is distributed under the terms of the Creative Commons Attribution License which permits any use, distribution, and reproduction in any medium, provided the original author(s) and the source are credited.

\section{References}

[1] Aczél, J.: Vorlesungen über Funktionalgleichungen und ihre Anwendungen. Birkhäuser, Basel (1961)

[2] Aczél, J., Chung, J.K., Ng, C.T.: Symmetric second differences in product form on groups. In: Rassias, Th.M. (ed.) Topics in mathematical analysis. Ser. Pure Math., vol. 11, pp. 1-22. World Scientific Publ. Co., Teaneck (1989)

[3] Aczél, J., Dhombres, J.: Functional equations in several variables. Cambridge University Press, London (1989)

[4] D'Alembert, J.: Mémoire sur les principes de mécanique. Hist. Acad. Sci., Paris, vol. 1769, pp 278-286

[5] Baker, J.A.: The stability of the cosine equation. Proc. Am. Math. Soc 80, 411$416(1980)$

[6] Chojnacki, W.: Group representations of bounded cosine functions. J. Reine Angew. Math. 478, 61-84 (1996)

[7] Corovei, I.: The extensions of the functions satisfying the cosine functional equation. Mathematica 18(41), 131-136 (1976)

[8] Corovei, I.: The cosine functional equation for nilpotent groups. Aequ. Math. I 5, 99-106 (1977)

[9] Deeba, E.Y., Koh, E.L.: D'Alembert functional equations in distributions. Proc. Am. Math. Soc. 116, 157-164 (1992)

[10] Dhombres, J., Ger, R.: Équations de Cauchy conditionnelles. C. R. Acad. Sc. Paris Sér. A 280, 513-515 (1975)

[11] Dhombres, J., Ger, R.: Conditional Cauchy equations. Glasnik Mat 13(33), 39-62 (1978)

[12] Friis, P.: D'Alembert's and Wilson's equations on Lie groups. Aequ. Math. 67, 12-25 (2004)

[13] Gajda, Z.: On functional equations associated with characters of unitary representations of groups. Aequ. Math. 44, 109-121 (1992) 
[14] Gessinger, A.: On the construction of cosine operator function and semigroups on function spaces with generator $a(x)\left(d^{2} / d x^{2}\right)+b(x)(d / d x)+c(x)$; theory. J. Comp. Anal. Appl. 3, 1-31 (2001)

[15] Gudder, S.: A generalization of d'Alembert's functional equation. Proc. Am. Math. Soc. 115, 419-425 (1992)

[16] Kannappan, P.: The functional equation $f(x y)+f\left(x y^{-1}\right)=2 f(x) f(y)$ for groups. Proc. Am. Math. Soc. 19, 69-74 (1968)

[17] Kannappan, P.: Cauchy equations and some of their applications. In: Rassias, Th.M. (ed.) Topics in mathematical analysis. Ser. Pure Math., vol. 11, pp. 518-538. World Scientific Publ. Co., Teaneck (1989)

[18] Ljubenova, E.T.: On d'Alembert's functional equation on an Abelian group. Aequ. Math. 22, 54-55 (1981)

[19] O'ConnorThomas, A.: A solution of d'Alembert's functional equation on a locally compact Abelian group. Aequ. Math. 15, 235-238 (1977)

[20] Penney, R.C., Rukhin, A.L.: D'Alembert's functional equation on groups. Proc. Am. Math. Soc. 77, 73-80 (1979)

[21] Stetkær, H.: D'Alembert's equation and spherical functions. Aequationes Math. 48, 220227 (1994)

[22] Stetkær, H.: D'Alembert's equation on metabelian groups. Aequ. Math. 59, 306-320 (2000)

[23] Stetkær, H.: D'Alembert's and Wilson's functional equation on step 2 nilpotent groups. Aequ. Math. 67, 241-262 (2004)

[24] Stetkær, H.: On operator-valued spherical functions. J. Funct. Anal. 224, 338-351 (2005)

[25] Szekélyhidi, L.: Almost periodicity and functional equations. Aequ. Math. 26, 163-175 (1983)

[26] Vasil'ev, V.V., Piskarev, S.I.: Differential equations in Banach spaces II. Theory of cosine operator functions. J. Math. Sci. 122, 3055-3174 (2004)

[27] Yang, D.: Factorization of cosine functions on compact connected groups. Math. Z. 254, 655-674 (2006)

Anna Bahyrycz and Janusz Brzdęk

Department of Mathematics

Pedagogical University

Podchorążych 2

30-084 Kraków

Poland

e-mail: bah@up.krakow.pl;

jbrzdek@up.krakow.pl

Received: February 3, 2012 